Localized diffusive motion on two different time scales in solid alkane nanoparticles

This article has been downloaded from IOPscience. Please scroll down to see the full text article.

2010 EPL 9166007

(http://iopscience.iop.org/0295-5075/91/6/66007)

View the table of contents for this issue, or go to the journal homepage for more

Download details:

IP Address: 128.206.162.204

The article was downloaded on 17/02/2011 at 19:22

Please note that terms and conditions apply. 


\title{
Localized diffusive motion on two different time scales in solid alkane nanoparticles
}

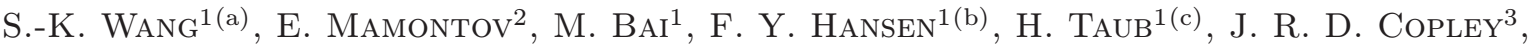 \\ V. García Sakai ${ }^{3,4(\mathrm{~d})}$, G. Gasparovic ${ }^{3}$, T. Jenkins ${ }^{3}$, M. Tyagi ${ }^{3,4}$, K. W. Herwig ${ }^{2}$, D. A. Neumann $^{3}$, \\ W. MontfrooiJ ${ }^{1}$ and U. G. VOLKMAnN $^{5}$ \\ ${ }^{1}$ Department of Physics and Astronomy and University of Missouri Research Reactor, University of Missouri \\ Columbia, MO 65211, USA \\ 2 Spallation Neutron Source, Oak Ridge National Laboratory - Oak Ridge, TN 37831, USA \\ ${ }^{3}$ Center for Neutron Research, National Institute of Standards and Technology - Gaithersburg, MD 20899-6102, USA \\ ${ }^{4}$ Department of Materials Science and Engineering, University of Maryland - College Park, MD 20742, USA \\ ${ }^{5}$ Facultad de Física, Pontificia Universidad Católica de Chile - Santiago, Chile
}

received 26 July 2010; accepted in final form 3 September 2010

published online 11 October 2010

PACS 66.30.hp - Molecular crystals

PACS 78.70.Nx - Neutron inelastic scattering

PACS 33.15.Mt - Rotation, vibration, and vibration-rotation constants

\begin{abstract}
High-energy-resolution quasielastic neutron scattering on three complementary spectrometers has been used to investigate molecular diffusive motion in solid nano- to bulk-sized particles of the alkane $n-\mathrm{C}_{32} \mathrm{H}_{66}$. The crystalline-to-plastic and plastic-to-fluid phase transition temperatures are observed to decrease as the particle size decreases. In all samples, localized molecular diffusive motion in the plastic phase occurs on two different time scales: a "fast" motion corresponding to uniaxial rotation about the long molecular axis; and a "slow" motion attributed to conformational changes of the molecule. Contrary to the conventional interpretation in bulk alkanes, the fast uniaxial rotation begins in the low-temperature crystalline phase.
\end{abstract}

Copyright (C) EPLA, 2010

It has long been known that molecular crystals can undergo one or more solid-solid phase transitions close to their melting point [1]. In the case of normal alkane molecules $\left(n-\mathrm{C}_{n} \mathrm{H}_{2 n+2}\right.$ hereafter denoted $\left.\mathrm{C} n\right)$, Müller studied their solid phases close to melting by X-ray diffraction nearly 80 years ago. He suggested that they might be characterized by quasi-free rotation of the molecular chains about their long axis but retain long-range positional order of the molecules in three dimensions [2]. The structure [3] and associated dynamics [4-6] of these so-called "rotator" phases has remained an active area of research as normal alkane chains are the basic building blocks of a wide variety of soft matter, including polymers, liquid crystals, and lipids [1]. More recently, interest in lubricants,

\footnotetext{
(a) Permanent address: Comprehensive Cancer Center - Palm Springs, CA 92263, USA.

(b) Permanent address: Department of Chemistry, Technical University of Denmark, IK 207 DTU - DK-2800 Lyngby, Denmark, EU.

(c) E-mail: taubh@missouri.edu

(d) Present address: ISIS Facility, Rutherford Appleton Laboratory Chilton, Didcot, OX11 0QX, UK, EU.
}

surfactants, and lipid membranes [1] has led to the investigation of rotator phases in restricted geometries such as alkane nanoparticles [7] and monolayer alkane films [8].

Despite intensive study, current understanding of the dynamics of the alkane rotator phases remains incomplete. There is, however, a general consensus that Müller's original rotator model [2] requires modification by including both translational diffusive motion parallel to the axis of rotation and intramolecular diffusive motion associated with the creation and annihilation of gauche defects in the alkane chains [5]. Measurements of the NMR second moment [9] have been useful in locating the solid-solid phase transitions occurring in proximity to the melting point and in examining models of the change in molecular motion at these transitions. However, these NMR measurements did not provide the time scale of the motion; so its identification has remained inconclusive. Ewen et al. conducted a comprehensive investigation by quasielastic neutron scattering (QENS) of uniaxially oriented bulk C33 samples [4,5]. They inferred that 
rotational motion about the long molecular axis and uncoupled translational motion parallel to this axis occur on a time scale of nanoseconds in the solid phase "C" just below the phase "D" closest to the melting point. In addition, a combination of structural characterization by X-ray scattering $[10,11]$ and optical spectra $[6,11]$ are consistent with the presence of gauche defects as originally proposed by Pechhold et al. [12] in the "D" phase immediately preceding the melting of bulk C33.

At this point, there remain several unanswered questions concerning the dynamics of the alkane plastic phases: 1) At what temperature do the various types of molecular diffusive motion begin and does their onset coincide with the observed structural phase transition(s)? 2) What experimental evidence is there for diffusive motion associated with conformational changes of the molecules and on what time scale does it occur? And 3) does the diffusive motion in the plastic phase depend on the size of the alkane particles and, if so, can these size effects help elucidate the type of diffusive motion?

Here we report a QENS investigation of the molecular diffusive motion in C32 particles of different sizes that addresses each of these questions. By using three complementary spectrometers, we are able to probe diffusive motion occurring on a time scale of $10^{-9}$ to $10^{-12} \mathrm{~s}$ and obtain the following principal results. We observe a systematic downward shift in both the crystalline-toplastic phase transition temperature and the melting point of the particles on reducing the particle size. For all particle sizes, we find that a relatively fast uniaxial rotation about the long molecular axis begins in the low-temperature crystalline phase rather than at the crystalline-to-plastic phase transition as has generally been interpreted. A slower diffusive motion associated with conformational changes of the molecules accompanies this uniaxial rotation. The number of molecules undergoing both types of motion is collectively enhanced at the crystalline-to-plastic phase transition.

In alkane films deposited from solution, one typically finds the topography shown in the Atomic Force Microscopy (AFM) image of fig. 1(a): partial layers in which the rod-shaped molecules are oriented with their long axis perpendicular to the $\mathrm{SiO}_{2}$ surface (labeled (2) and (3), the so-called perpendicular layers, coexisting with preferentially oriented nanoparticles (labeled (4)) [13]. Both the perpendicular layers and the nanoparticles reside on one or two layers of molecules aligned parallel to the $\mathrm{SiO}_{2}$ surface (the dark areas labeled (1), the so-called parallel layers. In the orthorhombic nanoparticles, the long axis of the C32 molecules is oriented perpendicular to the surface $[13,14]$. When these samples are thermally cycled in air above the bulk melting point, there is a tendency for the nanoparticles to grow at the expense of the partial perpendicular layers. This behavior is consistent with a Stranski-Krastanov growth mode at equilibrium in which preferentially oriented bulk particles nucleate above the parallel layers of the film [7].

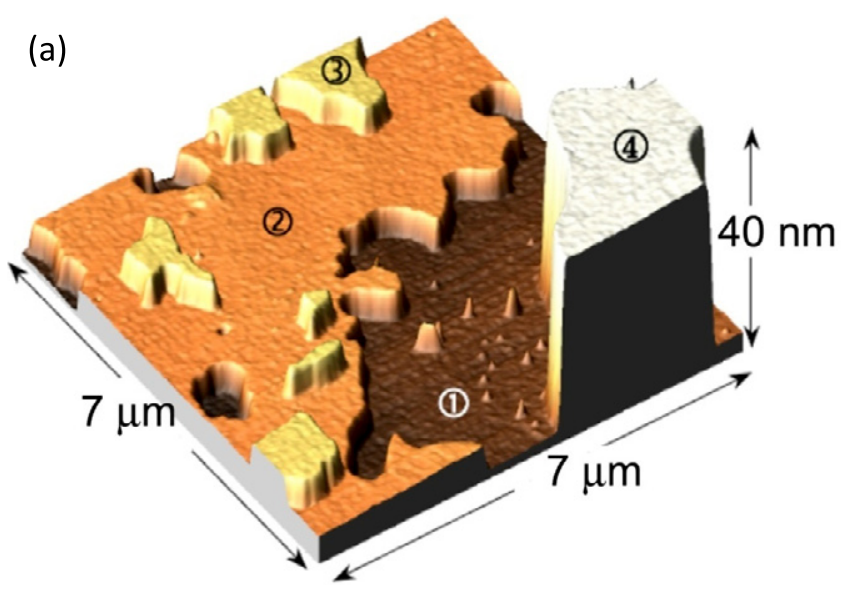

(b)

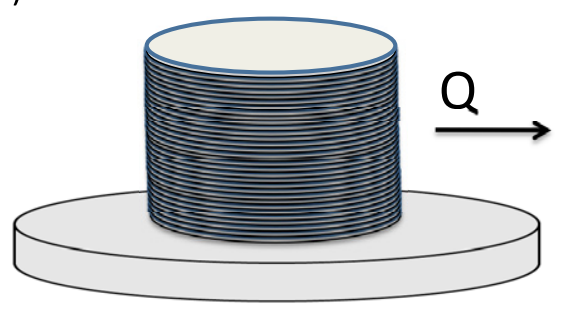

Fig. 1: (Colour on-line) (a) A 3D topographic image of a C32 film (ellipsometric thickness $\sim 94 \AA$ ) recorded by contactmode AFM (from ref. [13]). The dark areas (1) correspond to a visible portion of a complete layer of C32 molecules lying with their long axis parallel to the $\mathrm{SiO}_{2}$ surface. Residing on the parallel layer are an incomplete layer of perpendicular molecules (2), bilayer perpendicular islands (3), and a mesashaped nanoparticle (4)). (b) Schematic diagram of the neutron scattering sample consisting of a stack of $\mathrm{Si}(100)$ wafers and indicating the direction of the wave vector transfer $\mathbf{Q}=\mathbf{k}_{f}-\mathbf{k}_{i}$ with respect to the wafer plane.

Our samples of C32 nanoparticles were made by dipcoating an acid-cleaned, electronic-grade $\mathrm{Si}(100)$ wafer ( $5 \mathrm{~cm}$ diameter and $0.3 \mathrm{~mm}$ thick polished on both sides) in a solution of C32 dissolved in heptane (C7) [7,14]. The average particle size was controlled by varying the C32 concentration in the solution. The alkane purity was greater than 99.9\%. Each sample contained about 100 wafers forming a cylindrical stack and loaded into an aluminum cell sealed with an indium O-ring under an argon atmosphere (see fig. 1(b)). All the samples were heated at least $10 \mathrm{~K}$ above the C32 melting point (341 K) and then slowly cooled to $318 \mathrm{~K}$ where they were annealed for $20 \mathrm{~h}$ to form mesa-like particles on the surface having the surface-stabilized orthorhombic structure $[7,14]$. In this way, it was possible to make highly aligned orthorhombic nanoparticles with their $a-b$ plane oriented to within a few degrees of the scattering plane. Because the number of C32 molecules in the parallel layers adjacent to the $\mathrm{SiO}_{2}$ surface is estimated to be less than $1 \%$ of those in the perpendicular layers and nanoparticles above them, we neglect their contribution to the neutron spectra discussed below. 


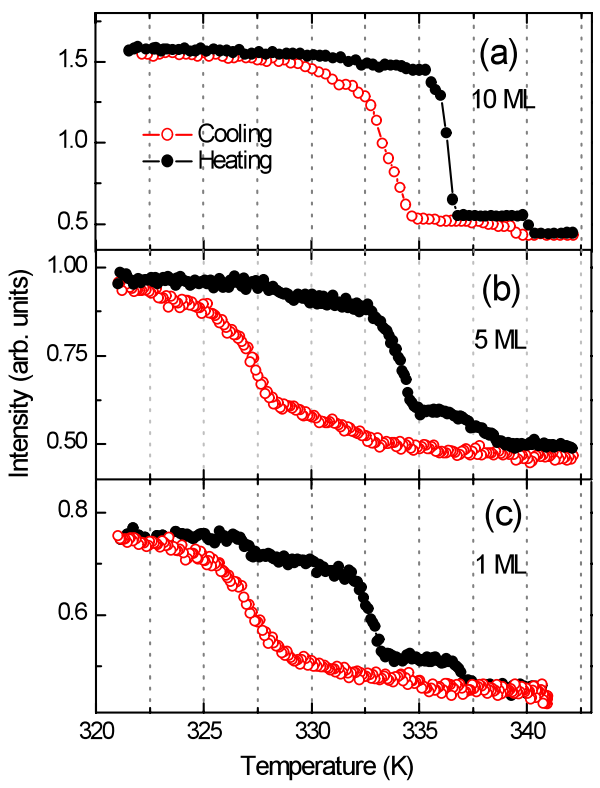

Fig. 2: (Colour on-line) Elastic intensity measured on heating (solid (black) points) and cooling (open (red) points) from C32 films of different coverages as measured on the backscattering spectrometer (HFBS) at NIST: (a) 10 ML (140-190 nm); (b) $5 \mathrm{ML}(60-90 \mathrm{~nm})$; and (c) $1 \mathrm{ML}(30-40 \mathrm{~nm})$. The range of particle heights in areas sampled by AFM on similarly prepared samples is indicated in parentheses. Error bars are comparable to the size of the plotting symbols in the figure.

We estimate the alkane film thickness in our neutron scattering experiments from AFM measurements of the total particle volume in a representative number of scan areas on similarly prepared samples. Using the $a$ and $b$ lattice constants of the orthorhombic particles [7], we express the total particle volume on a wafer surface as an equivalent number of layers (ML) in which the molecules are oriented perpendicular to the surface.

In fig. 2, we show the temperature dependence of the intensity of neutrons elastically scattered from samples of three different alkane coverages as measured on the highflux backscattering spectrometer (HFBS) at the NIST Center for Neutron Research [15]. The elastic intensity has been summed over all wave vector transfers and measures the number of neutrons scattered with energy transfers less than $\sim 1 \mu \mathrm{eV}$, the full width at half-maximum (FWHM) of the HFBS' resolution function. Incoherent scattering from the hydrogen atoms dominates the elastic signal so that a decrease in the elastic intensity measures the number of $\mathrm{H}$ atoms in the sample moving on a time scale faster than $\sim 1$ ns. Above the alkane film melting point, the translational and rotational diffusion of the C32 molecules results in unbounded motion of all $\mathrm{H}$ atoms on this faster time scale. Therefore, in its fluid phase, the C32 film no longer contributes to the elastic intensity; and one only observes elastic scattering from the silicon substrate.

On heating the $10 \mathrm{ML}$ sample in fig. 2(a), we see a step-like drop in intensity at a temperature of $\sim 337 \mathrm{~K}$ in agreement with the monoclinic-to-triclinic structural transition in bulk C32 as determined by X-ray diffraction [3] and ellipsometry [16]. A second downward step occurs at $\sim 340 \mathrm{~K}$, close to the melting point of bulk monoclinic C32 at $341 \mathrm{~K}$ [3]. As shown in fig. 2(a), these two steps reproduced on cooling, although with hysteresis indicating strongly first-order phase transitions. In the $5 \mathrm{ML}$ sample (fig. 2(b)), both of these sub steps are shifted a few degrees lower in temperature; and a new sub step appears below $330 \mathrm{~K}$. All three steps are shifted lower in temperature in the $1 \mathrm{ML}$ sample (fig. 2(c)) so that there is a downward sub step in intensity at $\sim 327 \mathrm{~K}$ followed by a second one at $\sim 332 \mathrm{~K}$ before all elastic intensity from the film is lost at $\sim 337 \mathrm{~K}$. By ramping the temperature at a slow rate of $0.05 \mathrm{~K} / \mathrm{min}$, we were able to reproduce the temperatures at which the intensity steps occurred.

AFM and synchrotron X-ray diffraction measurements [7] have shown that a nanoparticle of height $\sim 30-40 \mathrm{~nm}$ undergoes a solid-solid phase transition on heating to a temperature of $\sim 330 \mathrm{~K}$, i.e., $\sim 7 \mathrm{~K}$ below that in a monoclinic bulk particle [3]. Subsequent high-resolution ellipsometry measurements on C32 films gave evidence of a solid-solid transition at $\sim 332 \mathrm{~K}$ in multilayer islands of perpendicular molecules and one at $\sim 331 \mathrm{~K}$ in large monolayer islands of perpendicular molecules [16]. Therefore, we tentatively identify the two lowest-temperature sub steps in the elastic intensity of the $1 \mathrm{ML}$ sample (fig. 2(c)) with the solid-solid structural transition in monolayer and multilayer islands of perpendicular molecules, respectively. The disappearance of elastic scattering from the film at $\sim 337 \mathrm{~K}$ is consistent with the temperature at which a fluid monolayer phase is observed to spread outward from mesa-shaped nanoparticles on heating [7].

In order to determine the types of molecular motion responsible for the loss of elastic intensity in the C32 films on heating, we obtained quasielastic spectra on the newly operational backscattering spectrometer BASIS at the Spallation Neutron Source, Oak Ridge National Laboratory. BASIS, with its larger dynamic range $( \pm 100 \mu \mathrm{eV}$ at the time of our measurements), allows investigation of atomic motions that are up to a factor of three faster than can be investigated on the HFBS. This advantage of BASIS comes with only a small sacrifice in the slowest motions that can be observed as determined by its energy resolution function (FWHM $\sim 3.5 \mu \mathrm{eV}$ ). Moreover, BASIS has sufficient throughput that it is possible with our small samples to obtain quasielastic spectra at each of the data points in fig. 3 .

For our measurements on BASIS, we used the same $10 \mathrm{ML}$ sample whose elastic scan recorded on the HFBS appears in fig. 2(a). We first repeated the elastic scan as shown in fig. 3(a), again summed over all wave vector transfers, in order to verify sample quality and locate the steps in intensity marking the different phase transitions. Due to differences in thermometry in the cryostats used on the two spectrometers, we found it necessary to 


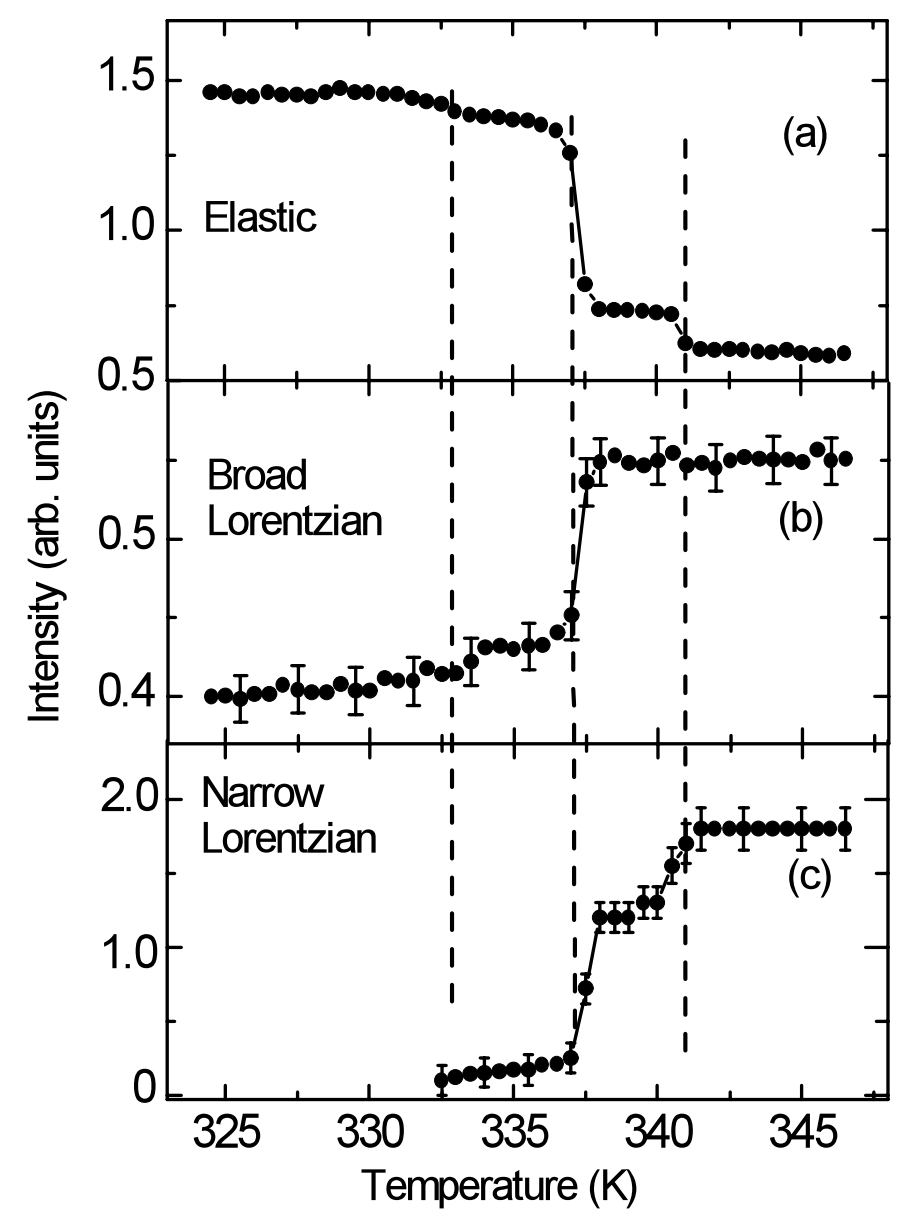

Fig. 3: Quasielastic scattered neutron intensity vs. temperature as measured on the $10 \mathrm{ML}$ sample, using the backscattering spectrometer (BASIS) at the SNS: (a) "elastic" intensity integrated over energy transfers spanning the instrumental resolution function. (b) Intensity measuring that of the "broad" Lorentzian fitted to the spectra and summed over all wave vector transfers (see text). For each spectrum (see fig. 4), the measured intensity is summed over a $20 \mu \mathrm{eV}$ band of energy transfers ending at $\pm 100 \mu \mathrm{eV}$ in order not to include intensity fit by the "narrow" Lorentzian. (c) Integrated intensity of the "narrow" Lorentzian summed over all $Q$.

correct the temperature scale to that shown in fig. 3 by requiring that the temperatures of the bulk melting point and the monoclinic-to-triclinic transition agree with those obtained on the HFBS. This recalibration was accomplished by shifting the temperatures measured in the BASIS cryostat downward by $2 \mathrm{~K}$.

We also observed a very weak step in the elastic intensity at $\sim 333 \mathrm{~K}$ that is difficult to identify in the HFBS elastic scan on this sample, but which corresponds to the largest step observed in the HFBS scan on the $1 \mathrm{ML}$ sample (fig. 2(c)). With the aid of ellipsometric measurements [16], we attribute this feature to multilayer islands of perpendicular molecules (see above). We conclude that the $10 \mathrm{ML}$ sample actually consists of bulk C32 particles coexisting with such multilayer islands, a topography consistent with elastic neutron diffraction patterns

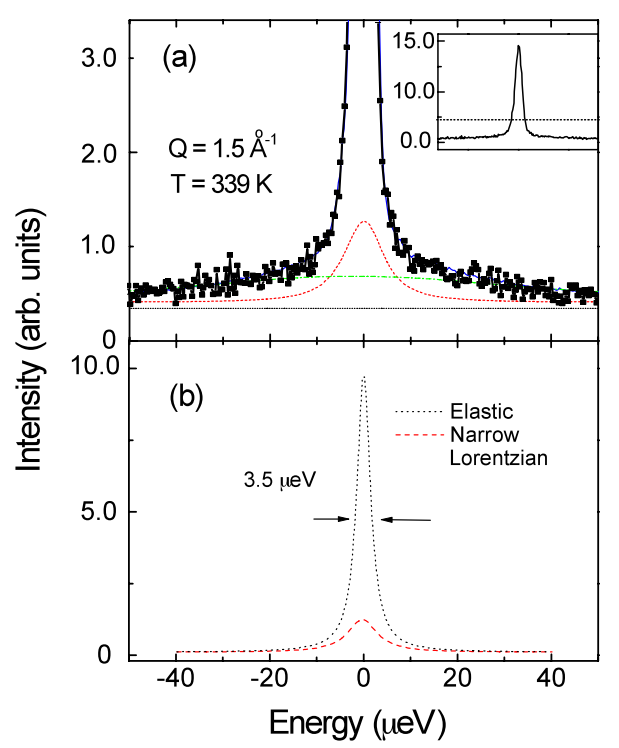

Fig. 4: (Colour on-line) (a) Typical spectrum measured on BASIS from the $10 \mathrm{ML}$ sample at $Q=1.5 \AA^{-1}$ and a temperature of $339 \mathrm{~K}$ at which the bulk particles in the film are in their plastic phase. The data points are filled squares. The spectrum has been fit to a scattering law composed of three terms: a delta function representing the elastic scattering plus two Lorentzians. The best fit shown by the solid (blue) line is obtained after folding each of these terms with the instrumental resolution function. It has three components: 1) the "broad" Lorentzian shown by the dash-dotted (green) line representing the rotation of the $\mathrm{C} 32$ molecules about their long axis; 2) the "narrow" Lorentzian $(F W H M=8.0 \mu \mathrm{eV})$ shown by the dashed (red) line which is identified with slower motions associated with conformational changes of the molecules; and 3) the elastic component shown by the dotted (gray) line in panel (b). The dotted (black) line indicates the background level. The full spectrum is shown in the inset where the data below the dotted line is that enlarged in panel (a). (b) Plot comparing the relative intensity of the narrow Lorentzian component of the spectrum shown by the dashed (red) line as in panel (a) with the elastic component having the same shape as the instrumental resolution function.

(not shown) taken on the triple-axis spectrometer at the University of Missouri Research Reactor.

A typical quasielastic spectrum measured on BASIS is shown in fig. 4(a) and corresponds to a temperature of $339 \mathrm{~K}$ and a wave vector transfer $Q=1.5 \AA^{-1}$. The wave vector transfer is aligned parallel to the plane of the silicon wafers (see fig. 1(b)) so that, with the preferred alignment of the C32 particles on the substrate, one is primarily sensitive to diffusive motion of the $\mathrm{H}$ atoms parallel to the $\mathrm{SiO}_{2}$ surface rather than parallel to the long axis of the C32 molecules. Recall that we do not have enough sensitivity to observe the $\mathrm{H}$-atom motion in the parallel layers adjacent to the $\mathrm{SiO}_{2}$ surface.

We fit the spectra by folding with the instrumental resolution function a scattering law composed of three terms: a delta function representing the elastic scattering plus two Lorentzians [17]. Utilizing the unprecedented 


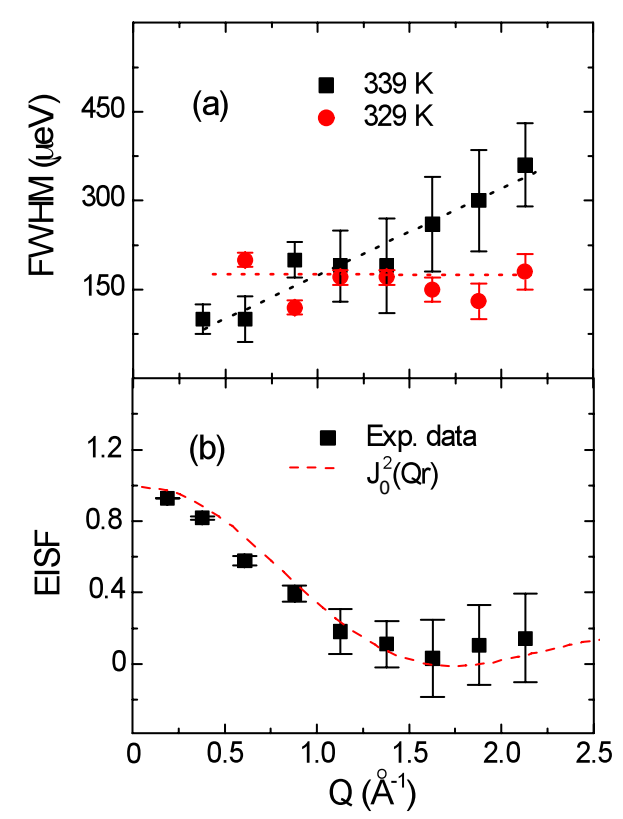

Fig. 5: (Colour on-line) (a) The FWHM of a single Lorentzian fit to quasielastic spectra measured from the $10 \mathrm{ML}$ sample using the DCS spectrometer at NIST. The bulk particles in the film are in their crystalline and plastic phases at $329 \mathrm{~K}$ and $339 \mathrm{~K}$, respectively. The dashed lines are guides to the eye. (b) Plot of the Elastic Incoherent Structure Factor (EISF) vs. $Q$ determined for the $10 \mathrm{ML}$ sample at $339 \mathrm{~K}$. The agreement with the function $J_{0}^{2}(Q r)$ with the value $r=1.41 \AA$ is consistent with uniaxial rotation about the long molecular axis. Note that the uncertainty in the EISF becomes large near its first node above $Q=1 \AA^{-1}$ where elastic scattering from the substrate dominates. The error bars result from a propagation of a standard deviation through the EISF calculation.

combination of energy resolution and dynamic range of BASIS, we can resolve two diffusive processes occurring at different rates in fits to the spectrum in fig. 4(a): a "slow" motion (time scale $\sim 0.5 \mathrm{~ns}$ ) described by a narrow Lorentzian (dashed curve) and a "fast" motion (time scale less than $\sim 50 \mathrm{ps)}$ that can be fit to a broad Lorentzian (dash-dotted curve). We tentatively associate the slower motion with conformational changes of the C32 molecules in analogy to the relatively slow intramolecular motion identified in similar quasielastic spectra of a C24 monolayer adsorbed on a graphite surface [8]. In fig. 4(b), we show that the intensity of the narrow Lorentzian is weak compared to the elastic component in the scattering; and its width $(\mathrm{FWHM}=8.0 \mu \mathrm{eV})$ is well beyond that of the resolution function $(\mathrm{FWHM}=3.5 \mu \mathrm{eV})$.

Because the broad Lorentzian in fig. 4(a) extends beyond the dynamic range of BASIS, it is preferable to analyze quasielastic spectra taken over a larger energy transfer range of $\pm 1 \mathrm{meV}$ obtained on the Disk Chopper Spectrometer (DCS) at NIST [18]. We fit the DCS spectra of the same $10 \mathrm{ML}$ sample in the low-temperature bulk crystalline phase at $329 \mathrm{~K}$ and in the bulk plastic phase at $339 \mathrm{~K}$ by folding the instrumental resolution function with a scattering law consisting of a delta function representing the elastic scattering plus a single Lorentzian (not shown) [17]. In fig. 5(a), we see that the FWHM of the Lorentzian in the crystalline phase at $329 \mathrm{~K}$ shows little wave vector transfer dependence characteristic of nontranslational diffusive motion. We tentatively identify this motion with uniaxial rotation about the long axis of the C32 molecules as will be examined below. The small $Q$-dependence of the FWHM in the plastic phase at $339 \mathrm{~K}$ (relative to that in the fluid phase) may be due to coupling of the uniaxial rotation to the "slow" motion associated with molecular conformational changes near the ends of the alkane chains [8].

At $339 \mathrm{~K}$, the Lorentzian intensity is large enough to determine the Elastic Incoherent Structure Factor (EISF), the fraction of the total scattering that is elastic, which gives the time-averaged spatial distribution of the proton motion [19]. To compute the EISF for our $10 \mathrm{ML}$ C32 film, we fit the DCS spectra at $339 \mathrm{~K}$ from our samples at each coverage as described above and then extrapolated the intensity of the elastic component to zero coverage to obtain the elastic scattering from the silicon substrate. It was subtracted to give the elastic intensity from the C32 film at each value of $Q$.

In fig. 5(b), we compare the measured EISF with the function $J_{0}^{2}(Q r)$ appropriate to uniaxial rotation of a C32 molecule about its long axis where $r$ is the radius of the methylene proton motion. We find a reasonable fit for a value $r=1.41 \AA$ corresponding to the all-trans configuration of the molecule. Note that the negative values and large uncertainty of the measured EISF near its first node are caused by the film's small elastic component and the imperfect subtraction of the elastic scattering from the silicon substrate. Also, conformational changes near the ends of the alkane chains associated with the "slow" intramolecular motion may cause some deviation from the EISF of an ideal uniaxial rotor, an effect that is difficult to estimate. We conclude that the broad Lorentzian is likely contributed by uniaxial rotation of a nearly all-trans C32 molecule on a time scale of $\sim 40 \mathrm{ps}$.

Having identified, both the narrow and broad Lorentzian contributions to the quasielastic scattering of the $10 \mathrm{ML}$ sample, it is of interest to consider the temperature dependence of their intensity as plotted in fig. 3. We see in (b) that the broad Lorentzian is already observable in the low-temperature crystalline phase and that its intensity increases gradually on heating until there is a step-like increase at $\sim 337 \mathrm{~K}$, the temperature of the bulk monoclinic-to-triclinic transition (cf. figs. 2(a) and $3(\mathrm{a})$ ). Therefore, for the bulk particles in the $10 \mathrm{ML}$ sample, the onset of uniaxial rotation is not at the transition to the so-called "rotator" phase as originally proposed [2]; but the number of molecules rotating is collectively enhanced at the monoclinic-to-triclinic transition presumably due to a decrease in the barrier to rotation. Using the DCS spectrometer at NIST, we have observed similar evidence of the uniaxial rotation 
beginning in the low-temperature crystalline phase of all samples in the 1 to $60 \mathrm{ML}$ coverage range. Moleculardynamics simulations confirm a gradual onset of hindered uniaxial rotation about the long molecular axis in multilayer films of perpendicular $\mathrm{C} 24$ molecules on a time scale of $\sim 20$ ps [20]. Furthermore, we note that a similar behavior occurs in monolayer films of intermediate-length alkanes oriented with their long axis parallel to the adsorbing surface. The onset of uniaxial rotation about the long molecular axis begins in the crystalline phase of C24 monolayers adsorbed on graphite and is enhanced at the crystalline-to-smectic phase transition [8,21].

In contrast, we find little or no enhancement of the uniaxial rotation at the solid-solid transition temperature at $\sim 333 \mathrm{~K}$ in fig. 3 (b) that we have identified as occurring in the multilayer islands of perpendicular molecules. However, the narrow Lorentzian becomes visible at this temperature and then undergoes a step-like increase in intensity at $\sim 337 \mathrm{~K}$, the temperature of the corresponding transition in the bulk particles (see fig. 3(c)). We attribute these features to enhanced proton motion associated with conformational changes of the molecules.

To summarize, our measurements using three complementary high-energy-resolution quasielastic neutron spectrometers reveal new features of the molecular diffusive motion associated with the long-studied solid-solid phase transitions near the melting point of alkane particles. As a model system, these results suggest that such a combined approach may be fruitful for dynamical studies of more complex polymers.

$$
* * *
$$

This work was supported by the U.S. National Science Foundation under Grant No. DMR-0705974 and utilized facilities supported in part by the NSF under agreement No. DMR-0454672. A portion of this research at Oak Ridge National Laboratory's Spallation Neutron Source was sponsored by the Scientific User Facilities Division, Office of Basic Energy Sciences, U.S. Department of Energy. We thank C. M. Brown for useful discussions.

\section{REFERENCES}

[1] Small D. M., The Physical Chemistry of Lipids: From Alkanes to Phospholipids (Plenum, New York) 1986.

[2] Müller A., Proc. R. Soc. A, 138 (1932) 514.
[3] Sirota E. B., King H. E jr., Singer D. M. and Shao Henry H., J. Chem. Phys., 98 (1993) 5809.

[4] Ewen B. and Richter D., J. Chem. Phys., 69 (1978) 2954.

[5] Ewen B., Strobl G. R. and Richter D., Faraday Discuss. Chem. Soc., 69 (1980) 19.

[6] Maroncelli M., Strauss H. L. and Snyder R. G., J. Chem. Phys., 82 (1985) 2811.

[7] Bai M., Knorr K., Simpson M. J., Trogisch S., Taub H., Ehrlich S. N., Mo H., Volkmann U. G. and Hansen F. Y., EPL, 79 (2007) 26003.

[8] Hansen F. Y., Criswell L., Fuhrmann D., Herwig K. W., Diama A., Dimeo R. M., Neumann D. A., Volkmann U. G. and Taub H., Phys. Rev. Lett., 92 (2004) 046103; Enevoldsen A. D., Hansen F. Y., Diama A., Taub H., Dimeo R. M., Neumann D. A. and Copley J. R. D., J. Chem. Phys., 126 (2007) 104704.

[9] Olf G. and Peterlin A., J. Polym. Sci. A-2, 8 (1970) $753 ; 771 ; 791$.

[10] Piesczek W., Strobl G. and Malzahn K., Acta Crystallogr. B, 30 (1974) 1278.

[11] Strobl G., Ewen B., Fischer E. W. and Piesczek W., J. Chem. Phys., 61 (1975) 5257.

[12] Pechiold W., Dollhopf W. and Engel A., Acustica, 17 (1966) 61.

[13] Trogisch S., Simpson M. J., Taub H., Volkmann U. G., Pino M. and Hansen F. Y., J. Chem. Phys., 123 (2005) 154703.

[14] Mo H., Taub H., Volkmann U. G., Pino M., Ehrlich S. N., Hansen F. Y., Lu E. and Miceli P., Chem. Phys. Lett., 377 (2003) 99.

[15] Mayer A., Dimeo R., Gehring P. and Neumann D., Rev. Sci. Instrum., 74 (2003) 2759.

[16] Cisternas E. A., Corrales T. P., Del Campo V., Soza P. A., Volkmann U. G., Bai M., Taub H. and Hansen F. Y., J. Chem. Phys., 131 (2009) 114705, 1-8.

[17] Azuah R. T., Kneller L. R., Qiu Y., TregennaPiggott P. L. W., Brown C. M., Copley J. R. D. and Dimeo R. M., J. Res. Natl. Inst. Stand. Technol., 114 (2009) 341.

[18] Copley J. R. D. and Cook J. C., Chem. Phys., 292 (2003) 477.

[19] Roe R.-J., Methods of X-ray and Neutron Scattering in Polymer Science (Oxford University Press, New York, Oxford) 2000.

[20] SozA P. A., PhD Thesis, Pontificia Universidad Católica de Chile (2009); more extensive simulations are in progress.

[21] Diama A., Matthies B., Herwig K. W., Hansen F. Y., Criswell L., Mo H., Bai M. and Taub H., J. Chem. Phys., 131 (2009) 084707. 